\title{
Highly Reliable Multilevel and 2-bit/cell Operation of Wrapped Select Gate (WSG) SONOS Memory
}

\author{
Woei-Cherng Wu, Tien-Sheng Chao, Wu-Chin Peng, Wen-Luh Yang, Jer-Chyi Wang, Jian-Hao Chen, \\ Chao-Sung Lai, Tsung-Yu Yang, Chien-Hsing Lee, Tsung-Min Hsieh, and Jhyy Cheng Liou
}

\begin{abstract}
In this letter, high-performance and reliable wrapped select gate (WSG) polysilicon-oxide-nitride-oxide-silicon (SONOS) memory cells with multilevel and 2-bit/cell operation have been successfully demonstrated. The multilevel storage is easily obtained with fast program/erase speed $(10 \mu \mathrm{s} / 5 \mathrm{~ms})$ and low programming current $(3.5 \mu \mathrm{A})$ for our WSG SONOS by a source-side injection. Besides the excellent reliability properties of our multilevel WSG-SONOS memory including unconsidered gate and drain disturbance, long charge retention $\left(>150{ }^{\circ} \mathrm{C}\right)$ and good endurance $\left(>10^{4}\right)$ are also presented. This novel WSG-SONOS memory with a multilevel and 2-bit/cell operation can be used in future high-density and high-performance memory application.
\end{abstract}

Index Terms-Multilevel operation, source-side injection, wrapped select gate (WSG) polysilicon-oxide-nitride-oxidesilicon (SONOS).

\section{INTRODUCTION}

$\mathbf{R}$ ECENTLY, the polysilicon-oxide-nitride-oxide-silicon (SONOS) memory device has received a lot of attention due to the advantages over the traditional floating-gate flash electrically erasable programmable read-only-memory device, including the reduced operation voltage [1], lower process complexity [2], elimination of drain-induced turn-ON [3], and improved cycling endurance [4]. To achieve the high-density flash memory device, multilevel storage and 2-bit/cell operation are becoming important topics, and it has been proposed that these flash devices [5]-[7] will be applied as the mass storage of portable handy terminals, solid-state cameras, and PC cards. By storing different amount of charges in the nitride trapping layers to reliably distinguish different levels and treating these levels as different combination of bits, the multilevel storage can be successfully performed. In addition, the 2-bit/cell op-

Manuscript received October 19, 2006; revised December 13, 2006. This work was supported in part by the National Science Council, Taiwan, R.O.C., under Contract NSC-95-2221-E-009-272, and in part by MOE-ATU. The review of this letter was arranged by Editor Y. Taur.

W.-C. Wu, T.-S. Chao, and T.-Y. Yang are with the Department of Electrophysics, National Chiao Tung University, Hsinchu 300, Taiwan, R.O.C. (e-mail: tschao@mail.nctu.edu.tw).

W.-C. Peng and W.-L. Yang are with the Department of Electronic Engineering, Feng Chia University, Taichung 40724, Taiwan R.O.C.

J.-C. Wang is with the Nanya Technology Corporation, Taoyuan 333, Taiwan, R.O.C.

J.-H. Chen is with the Department of Electronic Engineering, National Chiao-Tung University, Hsinchu 300, Taiwan, R.O.C.

C.-S. Lai is with the Department of Electronic Engineering, Chang Gung University, Kwei-Shan, Taoyuan 333, Taiwan, R.O.C.

C.-H. Lee, T.-M. Hsieh, and J. C. Liou are with the Solid State System Corporation, Hsinchu 302, Taiwan, R.O.C

Digital Object Identifier 10.1109/LED.2007.891301 eration for a single cell by using the forward/reverse reading method also effectively enhanced the performance of SONOS memory device. Nevertheless, owing to some critical issues such as charge redistribution during the program [8], charge diffusion in silicon nitride after injection [9], and bottom oxide charge by the cycling [10], it will be more difficult to obtain the local 2-bit/cell characteristics and multilevel operation in sub-90-nm regime. Besides, the multilevel operation is hard to perform by the conventional channel hot electron injection and Fowler-Nordheim tunneling with the low efficiency of the electron injection, poor programming speed, and high operation voltage. Consequently, a novel source-side injection technique has been proposed in the SONOS memory for high-speed programming [11]-[13]. In this letter, we for the first time demonstrate that the wrapped select gate (WSG) SONOS memory with the source-side injection exhibits highperformance multilevel and 2-bit/cell operation. A fast and excellent control of the storage charge is obtained even after large programming disturbance and $>10^{4}$ program/erase $(\mathrm{P} / \mathrm{E})$ cycling. Moreover, a superior charge retention behavior after a high-temperature baking $\left(>150{ }^{\circ} \mathrm{C}\right)$ of the WSG-SONOS memory is also presented for highly reliable multilevel and 2-bit/cell flash application.

\section{EXPERIMENTAL}

The simple cross-sectional structure of the WSG-SONOS device was indicated in Fig. 1 , and the $0.18-\mu \mathrm{m}$ ground rule technology was used to fabricate our device. The select gate, which was used for an assist gate in the source-side injection, is wrapped with both an oxide-nitride-oxide layer and a word line gate. The fabrication procedures were therefore easily compatible with the general process for the fabrication of complementary metal-oxide-semiconductor integrated circuit. Besides, both source and drain regions can be used as the bit line in the WSG-SONOS memory. In our device, the tunneling oxide/nitride/blocking oxide layers were 5.0/8.0/10.0-nm thick, and the length and width of the select gate were 0.18 and $0.36 \mu \mathrm{m}$, respectively.

According to our WSG-SONOS structure, the 2-bit/cell operation of the WSG-SONOS memory can be easily achieved, as shown in the inset of Fig. 1. In addition, the basic twobit operation conditions of the WSG-SONOS memory were listed in the inset table. Here, only bit-1 was programmed and bit-2 was on initial state. A clear two physical bits storing characteristic in our WSG-SONOS memory was demonstrated from the threshold voltage difference of these two bits which 


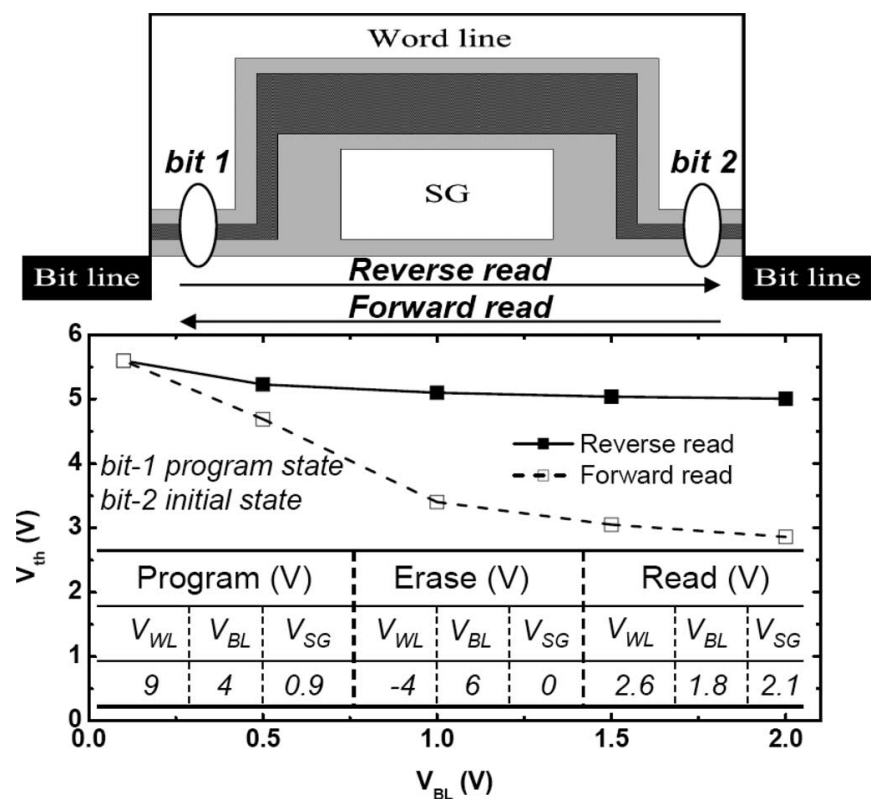

Fig. 1. Cross-sectional scheme of a 2-bit/cell WSG-SONOS memory device with WSG structure. The inset figure shows the excellent 2-bit/cell characteristics for the WSG-SONOS memory. In addition, the basic operation conditions of WSG-SONOS memory are listed in the inset table.

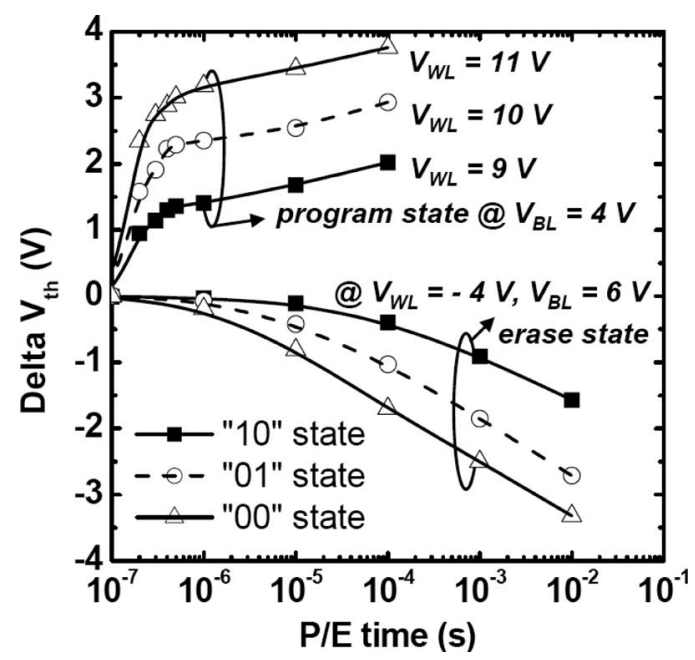

Fig. 2. Multilevel programming and erasing characteristics. The $V_{\text {th }}$ shift is larger than 1,2 , and $3 \mathrm{~V}$ at $V_{\mathrm{SG}}=0.9 \mathrm{~V}$, while the programming time is only $1 \mu \mathrm{s}$ for $V_{\mathrm{WL}}=9,10$, and $11 \mathrm{~V}$, respectively.

can reach up to $2.0 \mathrm{~V}$ during reading, while the drain and source were biased at 1.8 and $0 \mathrm{~V}$, respectively.

\section{RESULTS AND DISCUSSION}

To achieve the multilevel operation, 9, 10, and $11 \mathrm{~V}$ were applied to the word gate while the drain, source, and select gate were biased at 4,0 , and $0.9 \mathrm{~V}$, respectively. Under this bias condition, the WSG-SONOS memory was programmed by the source-side injection mechanism. Therefore, as shown in Fig. 2, the word line biased at 9, 10, and $11 \mathrm{~V}$ to achieve the "10," " 01 ," and " 00 " states (programming time $T_{p}$ is $1 \mu \mathrm{s}$ ) can be easily obtained, while the device is reverse-read at $V_{\mathrm{BL}}=1.8 \mathrm{~V}$. The window from state to state is larger than
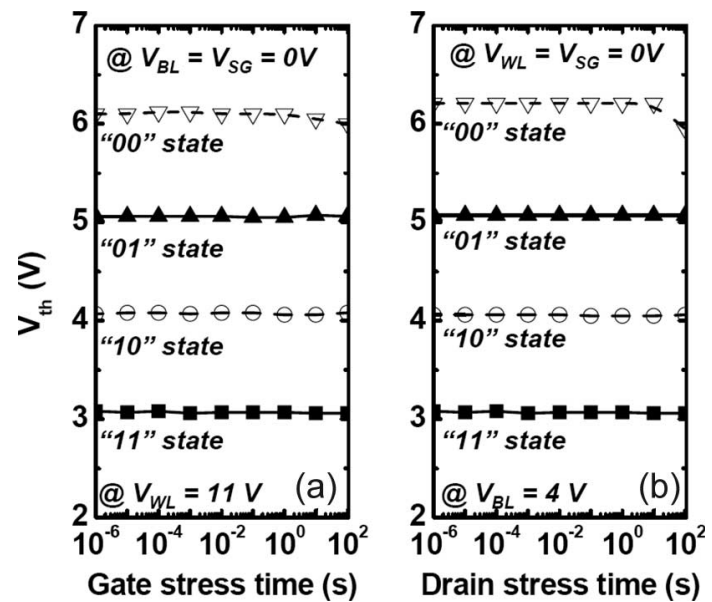

Fig. 3. (a) Gate and (b) drain disturbance performance of the WSG-SONOS memory for the multilevel operation. The $V_{\text {th }}$ shift is only about $0.1 \mathrm{~V}$ after 100 -s word-line stressing $\left(V_{\mathrm{WL}}=11 \mathrm{~V}\right)$ for the " 00 " state and $0.5 \mathrm{~V}$ after 100 -s bit-line stressing $\left(V_{\mathrm{BL}}=4 \mathrm{~V}\right)$ for the " 00 " state.

$1 \mathrm{~V}$ for programming at $V_{\mathrm{SG}}=0.9 \mathrm{~V}$, and $T_{p}$ is only $1 \mu \mathrm{s}$. On the other hand, with the erasing time of only $5 \mathrm{~ms}$ for $V_{\mathrm{WL}}=-4 \mathrm{~V}$ and $V_{\mathrm{BL}}=6 \mathrm{~V}$, the multilevel bits can be easily erased by a band-to-band hot hole erasing. Besides, the high programming speed is achieved by quite low programming current smaller than $3.5 \mu \mathrm{A}$ (" 00 " state). We believe that this highspeed programming performance of the multilevel operation is attributed to the high-injection efficiency of the source-side injection mechanism [11].

The failure phenomenon, i.e., "program disturbance," often takes place under the electrical stress applied to those neighboring unprogrammed cells during programming a specific cell in the array. Two types of program disturbances including gate (word-line) disturbance and drain/source (bit-line) disturbance are needed to be considered. Fig. 3(a) and (b) shows the gate and drain stressed characteristics of the WSG-SONOS memory with the multilevel operation, respectively. The deviation by the gate disturbance can be found to be less than $0.1 \mathrm{~V}$ after 100-s stress for all states. On the other hand, only a small negative shift in " 00 " state (initial state) of the drain disturbance is observed due to the drain field induced hole injection by a bandto-band tunneling [14]. This drain disturbance can be almost ignored for the other multilevel states of our WSG-SONOS memory device.

Fig. 4 shows the data retention behavior of the WSG-SONOS memory with a multilevel operation at the elevated temperatures of $85^{\circ} \mathrm{C}, 150^{\circ} \mathrm{C}$, and $250{ }^{\circ} \mathrm{C}$, respectively. There is almost no charge loss for the multilevel operation of WSG-SONOS memory at $85{ }^{\circ} \mathrm{C}$ and $150{ }^{\circ} \mathrm{C}$, as indicated in this figure. However, a slightly increased charge loss is observed under the quite high-temperature condition of $250{ }^{\circ} \mathrm{C}$. This charge loss may be resulted from the detrapping process in which the trapped electrons tend to migrate and redistribute in the silicon nitride at elevated temperature [14]. However, this charge loss saturates, and the different states of multilevel operation can still easily be discerned, as shown in this figure. Furthermore, the endurance characteristic of the WSG-SONOS memory is shown in Fig. 5. The cell programmed at $V_{\mathrm{WL}}=9,10$, and 


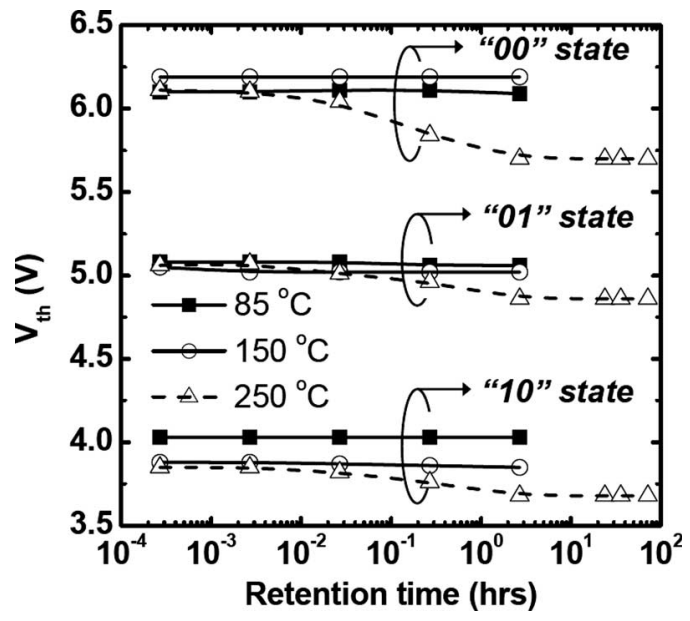

Fig. 4. Data retention performance of the WSG-SONOS memory for the multilevel operation after different high-temperature baking. The $V_{\mathrm{th}}$ shift for " 00 " state is smaller than $0.5 \mathrm{~V}$ after $10000 \mathrm{~s}$ at $250^{\circ} \mathrm{C}$ baking.

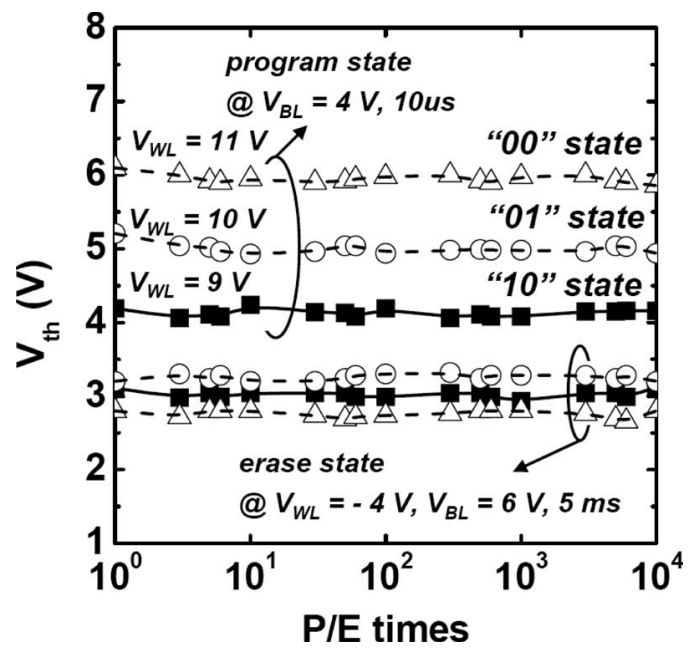

Fig. 5. Endurance characteristics of the WSG-SONOS memory for the multilevel operation. The memory window is almost the same even after $10 \mathrm{k} \mathrm{P} / \mathrm{E}$ cycling for the different programming states.

$11 \mathrm{~V}$, and $V_{\mathrm{BL}}=4 \mathrm{~V}$ for $10 \mu \mathrm{s}$, and erased at $V_{\mathrm{WL}}=-4 \mathrm{~V}$ and $V_{\mathrm{BL}}=6 \mathrm{~V}$ for $5 \mathrm{~ms}$ was used for multilevel operation. The threshold voltage of each state is almost the same even after $10^{4} \mathrm{P} / \mathrm{E}$ cycles. Nearly negligible window narrowing for multilevel operation of the WSG-SONOS memory is observed, meaning that the high-performance and reliable flash memory is successfully realized.

\section{CONCLUSION}

For the first time, a novel and highly reliable multilevel and 2-bit/cell WSG-SONOS memory was demonstrated. The multilevel storage is easily obtained with a fast P/E speed $(10 \mu \mathrm{s} / 5 \mathrm{~ms})$ by the source-side injection. In addition, the gate and drain disturbances for multilevel storage of this memory can be nearly ignored. The superior data retention $\left(>150{ }^{\circ} \mathrm{C}\right)$ and endurance characteristics $\left(10^{4}\right)$ indicated that this WSGSONOS memory with multilevel and 2-bit/cell operation can be applied into future high-density and high-performance flash memory.

\section{ACKNOWLEDGMENT}

The authors would like to thank the Solid State System Co., Ltd. for providing samples.

\section{REFERENCES}

[1] M. White, Y. Yang, P. Ansha, and M. L. French, "A low voltage SONOS nonvolatile semiconductor memory technology," IEEE Trans. Compon., Packag., Manuf. Technol., vol. 20, no. 2, pp. 190-195, Jun. 1997.

[2] T. Y. Chan, K. K. Young, and C. Hu, "A true single-transistor oxidenitride-oxide EEPROM device," IEEE Electron Device Lett., vol. EDL-8, no. 3, pp. 93-95, Mar. 1987.

[3] M. K. Cho and D. M. Kim, "High performance SONOS memory cells free of drain turn-on and over-erase: Compatibility issue with current flash technology," IEEE Electron Device Lett., vol. 21, no. 8, pp. 399-401, Aug. 2000.

[4] M. She, H. Takeuchi, and T. J. King, "Silicon-nitride as a tunnel dielectric for improved SONOS-type flash memory," IEEE Electron Device Lett., vol. 24, no. 5, pp. 309-311, May 2003.

[5] M. Specht, U. Dorda, L. Dreeskomfeld, J. Kretz, F. Hofmann, M. Stadele, R. J. Luyken, W. Rosner, H. Reisinger, E. Landgraf, T. Schulz, J. Hartwich, R. Kommling, and L. Risch, "20 nm tri-gate SONOS memory cells with multi-level operation," in IEDM Tech. Dig., 2004, pp. 1083-1085.

[6] S. S. Chun, C. M. Yih, S.T. Liaw, Z. H. Ho, S. S. Wu, C. J. Lin, D. S. Kuo, and M. S. Liang, "A novel high performance and reliability p-type floating gate N-channel flash EEPROM," in VLSI Symp. Tech. Dig., 1999, pp. 19-20.

[7] W. J. Tsai, N. K. Zous, T. Wang, Y. H. Joseph Ku, and C. Y. Lu, "A novel operation method to avoid overerasure in a scaled trapping-nitride localized charge storage flash memory cell and its application for multilevel programming," IEEE Trans. Electron Devices, vol. 53, no. 4, pp. 808814, Apr. 2006.

[8] Y. K. Lee, T. H. Kim, S. H. Lee, J. D. Lee, and B. G. Park, "Twin-bit silicon-oxide-nitride-oxide-silicon (SONOS) memory by inverted sidewall patterning (TSM-ISP)," in Proc. IEEE Dig. Silicon Nanoelectron. Workshop, 2003, pp. 92-93.

[9] E. Lusky, Y. Shacham-Diamand, I. Bloom, and B. Eitan, "Electrons retention model for localized charge in oxide-nitride-oxide (ONO) dielectric," IEEE Electron Device Lett., vol. 23, no. 9, pp. 556-558, Sep. 2002.

[10] W. J. Tsai, N. K. Zous, C. J. Lie, C. C. Liu, C. H. Chen, and T. Wang, "Data retention behavior of a SONOS type two-bit storage flash memory cell," in IEDM Tech. Dig., 2001, pp. 719-722.

[11] H. Tomyie, T. Terano, K. Nomoto, and T. Kobayashi, "A novel 2-bit/cell MONOS memory device with a wrapped-control-gate structure that applies source-side hot electron injection," in VLSI Symp. Tech. Dig., 2002, pp. 206-207.

[12] K. T. Chang, W. M. Chen, C. Swift, J. M. Higman, W. M. Paulson, and K. M. Chang, "A new SONOS memory using source-side injection for programming," IEEE Electron Device Lett., vol. 19, no. 7, pp. 253-255, Jul. 1998.

[13] T. Kawamura, Y. Sasago, H. Kurata, K. Otsuga, S. Noda, K. Kozakai, and T. Kobayashi, "Negative-source enhanced source-side injection achieving 100-ns cell programming in multilevel flash memories," in VLSI Symp. Tech. Dig., 2005, pp. 206-207.

[14] C. S. Hsieh, P. C. Kao, C. S. Chiu, C. H. Hon, C. C. Fan, W. C. Kung, Z. W. Wang, and E. S. Jeng, "NVM characteristics of single-MOSFET cells using nitride spacers with gate-to-drain NOI," IEEE Trans. Electron Devices, vol. 51, no. 11, pp. 1811-1817, Nov. 2004. 